\title{
Editorial DYNAMIC EQUATIONS AND APPLICATIONS
}

\author{
MARTIN BOHNER AND ALLAN PETERSON
}

Received 20 May 2006; Accepted 20 May 2006

Dedicated to Professor Gusein Guseinov on the occasion of his 55th birthday

Copyright (c) 2006 M. Bohner and A. Peterson. This is an open access article distributed under the Creative Commons Attribution License, which permits unrestricted use, distribution, and reproduction in any medium, provided the original work is properly cited.

Going back to its founder Stefan Hilger (1988), the study of dynamic equations on time scales is a fairly new area of mathematics. Motivating the subject is the notion that dynamic equations on time scales can build bridges between continuous and discrete mathematics. Time is considered to be an element of an arbitrary closed subset of the reals, the so-called time scale. Dynamic equations on the time scale of all real numbers are differential equations, while dynamic equations on the time scale of all integers are difference equations. But not only is this theory able to unify the continuous and the discrete, it also can help to extend these theories to cases "in between" and to other dynamic equations (e.g., q-difference equations). The study of time scales theory has led to several important applications, for example, in the study of insect population models, neural networks, heat transfer, quantum mechanics, and epidemic models.

This special issue on Dynamic Equations and Applications features fifteen articles by some of the top researchers in time scales worldwide. The impressive list of twenty-five contributors from eight different countries includes Elvan Akın-Bohner (USA), Douglas Anderson (USA), Mouffak Benchohra (Algeria), Martin Bohner (USA), Alberto Cabada (Spain), Fordyce Davidson (United Kingdom), Lynn Erbe (USA), Nicole Garbers (Germany), Gusein Guseinov (Turkey), Samira Hamani (Algeria), Johnny Henderson (USA), Gro Hovhannisyan (USA), Basant Karna (USA), Peter Kloeden (Germany), Bonita Lawrence (USA), Allan Peterson (USA), Christian Pötzsche (USA), Youssef Raffoul (USA), Pavel Řehák (Czech Republic), Andreas Ruffing (Germany), Bryan Rynne (United Kingdom), Yeter Şahiner (Turkey), Samir Saker (Egypt), Petr Stehlík (Czech Republic), and Alexandra Zmorzynska (Germany).

We would like to dedicate this special issue to Professor Gusein Guseinov on the occasion of his 55th birthday. Professor Guseinov received his Ph.D. degree from Moscow State University in 1977 and was Head of the Department "Spectral Theory of Operators" at the Azerbaijan Academy of Sciences before joining Ege University (Izmir, Turkey) in 1993 and Atilim University (Ankara, Turkey) in 2001. 